\title{
Associação entre a doença periodontal e a doença pulmonar obstrutiva crônica: uma revisão de literatura
}

\author{
Association between periodontal disease and chronic obstructive \\ pulmonary disease: a literature review
}

\section{Cristiana da Costa Libório-Lago', Fernanda Warken Rosa Camelier', Carina Oliveira dos Santos ${ }^{3}$, Marcio Ramos Coutinho ${ }^{4}$, Sandro Bittencourt ${ }^{5}$, Aquiles Assunção Camelier ${ }^{6}$}

'Autora para correspondência. Escola Bahiana de Medicina e Saúde Pública (EBMSP), ${ }^{2}$ Universidade do Estado da Bahia (UNEB), Salvador, Bahia, Brasil. ORCID: 0000-0003-2540-0142. fcamelier@uneb.br ${ }^{3}$ Escola Bahiana de Medicina e Saúde Pública (EBMSP), Universidade do Estado da Bahia (UNEB), Salvador, Bahia, Brasil. ORCID: 0000-0003-1367-9326.crods@bahiana.edu.br ${ }^{4}$ Universidade do Estado da Bahia (UNEB), Universidade Salvador (UNIFACS), Salvador, Bahia, Brasil. ORCID: 0000-0003-4537-0681.marcoutinho@uneb.br ${ }^{5}$ Escola Bahiana de Medicina e Saúde Pública (EBMSP), Salvador - Bahia, Brasil. ORCID: 0000-0003-4990-8215. sandrobittencourt@bahiana.edu.br ${ }^{\circ}$ Escola Bahiana de Medicina e Saúde Pública (EBMSP), Universidade do Estado da Bahia (UNEB), Salvador, Bahia, Brasil. ORCID: 0000-0001-5410-5180. aquilescamelier@yahoo.com.br

RESUMO | OBJETIVO: realizar uma revisão acerca da associação da doença periodontal com a Doença Pulmonar Obstrutiva Crônica (DPOC). METODOLOGIA E RESULTADOS: Trata-se de um estudo de revisão de literatura. Os descritores em português foram selecionados no DeCS (BVS), "doença periodontal", "doença pulmonar obstrutiva crônica" e "periodontite crônica", e em inglês, no $\mathrm{MeSH}$, "periodontal disease", "chronic obstructive pulmonary disease" e "chronic periodontitis". A busca foi realizada nas bases de dados MEDLINE, LILACS e SciELO, através dos portais PubMed, Capes e BVS, no período de 1999 a 2018 . Foram selecionados 49 artigos e o relatório de consenso elaborado pela Iniciativa Global para DPOC (GOLD). CONCLUSÕES: A DPOC é a quarta causa de morte no mundo e apesar do número crescente de publicações, poucos estudos foram realizados na América do Sul. Os estudos mostram que a DPOC e a doença periodontal possuem fatores de risco e comorbidades em comum e cuja associação pode acontecer pela presença de bactérias na cavidade oral (aspiradas ou via corrente sanguínea) e por serem, ambas, doenças inflamatórias de caráter essencialmente neutrofílico. A maioria dos estudos evidenciou piores índices periodontais nos pacientes com DPOC, associando-se a uma maior frequência de exacerbações. Por outro lado, a DPOC também pode ser um fator de risco para o aumento da severidade da doença periodontal. Desta forma, a introdução do tratamento periodontal e ações educativas para promoção de saúde bucal no protocolo de acompanhamento da DPOC pode propiciar maior abrangência e resolutividade no tratamento destes pacientes.

PALAVRAS-CHAVE: Doença periodontal, doença pulmonar obstrutiva crônica, periodontite crônica, saúde bucal, doenças respiratórias.

\begin{abstract}
I AIM: to review the association between periodontal disease and Chronic Obstructive Pulmonary Disease (COPD). METHODS AND RESULTS: This is a literature review study. The descriptors in Portuguese were selected from DeCS (BVS), "doença periodontal", "doença pulmonar obstrutiva crônica" and "periodontite crônica" and, in English, from MeSH, "periodontal disease", "chronic obstructive pulmonary disease" and "periodontitis ". The search was performed in the MEDLINE, LILACS and SciELO data sources through the PubMed, Capes and BVS portals, from 1999 to 2018 . It was selected 49 articles and the consensus from the Global Initiative for Chronic Obstructive Lung Disease (GOLD).

CONCLUSIONS: COPD is the fourth leading cause of death in the world and despite the increasing number of publications, few studies have been conducted in South America. Studies show that COPD and periodontal disease have common risk factors and comorbidities and whose association may be due to the presence of oral bacteria (aspirated or through the bloodstream) and because both diseases are neutrophilic inflammatory conditions. Most studies have shown worse periodontal indices in patients with COPD, and these findings were associated with a higher frequency of exacerbations. On the other hand, COPD may also be a risk factor for increasing the severity of periodontal disease. These literature evidences suggest the introduction of periodontal treatment and educational actions for prevention and promotion of oral health as part of COPD patient's follow-up to improve resolution and effectiveness of COPD treatment.
\end{abstract}

KEYWORDS: Periodontal disease, chronic obstructive pulmonary disease, chronic periodontitis, oral health, respiration disorders. 
Introdução

Tanto a Doença Pulmonar Obstrutiva Crônica (DPOC) como a doença periodontal são patologias inflamatórias crônicas, cuja associação tem sido alvo de crescente interesse, embora ainda não tenha sido completamente elucidada. A resposta inflamatória é um processo fisiológico protetor que tem por finalidade minimizar ou eliminar o efeito prejudicial de um mecanismo e/ou agente agressor. A resolução da inflamação constitui uma sequência de eventos realizados por mediadores químicos. Se alguma falha acontece na resolução deste quadro, uma patologia crônica se instala'.

A DPOC é resultante de um processo inflamatório que provoca obstrução crônica e recorrente ao fluxo de $a r^{2}$. Embora o tabagismo seja o principal fator de risco, a DPOC decorre também de uma comple$x a$ interação entre diversos outros fatores. A heterogeneidade clínica e fenotípica da DPOC está ainda associada a uma diversidade de comorbidades, dentre as quais está a doença periodontal ${ }^{2,3,4}$.

A doença periodontal é também um problema de saúde pública que atinge mais da metade da população mundial, nos estágios leve e moderado, e cerca de $5 \%$ a $20 \%$, na sua forma severa ${ }^{5}$. Caracteriza-se por um processo crônico inflamatório resultante da perda da homeostase microbiana acumulada no biofilme, que pode levar a perda dos tecidos de suporte e da própria unidade dentária' ${ }^{1,6,7}$. A doença periodontal estabelece potencial associação com um número grande de comorbidades, como doença cardiovascular, diabetes, artrite reumatóide, osteoporose, doenças neurológicas e respiratórias ${ }^{8}$.

A associação entre a DPOC e a doença periodontal pode acontecer por mecanismos relacionados à aspiração de bactérias patogênicas da cavidade oral ${ }^{4,9,10}$ e ao aumento das citocinas inflamatórias na corrente sanguínea, com os neutrófilos desempenhando um papel chave neste processo ${ }^{4,11}$. O impacto dessa associação é proposto como uma relação bidirecional: a hipótese de que um agravamento do curso clínico ${ }^{12,13}$ e do risco ${ }^{14-19}$ de desenvolver a DPOC pode ser provocado pela doença periodontal, como também a de que um agravamento da doença periodontal pode ser provocado pela $D_{P O C}{ }^{20,21}$. Influenciando esta associação, a DPOC e a doença periodontal possuem muitos fatores de risco em comum, como o tabagismo, idade, nível de escolaridade, status socioeconômico e aspectos comportamentais relacionados ao autocuidado ${ }^{4,22}$.

Recentemente tem havido um interesse crescente na melhor compreensão desta associação e suas implicações, a fim de aprimorar a resolutividade do acompanhamento e tratamento dos pacientes com DPOC. O tratamento periodontal e a educação em saúde bucal voltada para as pacientes de alto risco resultam em economias significativas de custo e melhoria da qualidade de vida para estes pacientes ${ }^{14,17,24,25}$. Desta forma, este estudo teve como objetivo realizar uma revisão de literatura acerca da associação da doença periodontal com a DPOC.

\section{Metodologia}

Trata-se de um estudo de revisão de literatura do tipo descritivo, a partir de publicações acerca da associação entre a doença periodontal e a doença pulmonar obstrutiva crônica.

Após a definição do tema e objetivo deste estudo, os descritores em inglês foram selecionados no MeSH (Medical Subject Headings) e em português no DeCS (Descritores em Ciências da Saúde) da BVS (Biblioteca Virtual em Saúde), determinando-se os seguintes termos: periodontal disease/doença periodontal, chronic obstructive pulmonary disease/ doença pulmonar obstrutiva crônica, chronic periodontitis/periodontite crônica.

A pesquisa eletrônica foi então realizada utilizando-se os descritores nos portais PubMed (Medline Público), portal de periódicos CAPES (Coordenação de Aperfeiçoamento de Pessoal de Nível Superior) e no BVS, para busca nas bases de dados MEDLINE (Sistema Online de Busca e Análise de Literatura Médica), LILACS (Literatura Latino-Americana e do Caribe em Ciências da Saúde) e SciELO (Biblioteca Eletrônica Científica Online).

Sobre as publicações disponibilizadas, após leitura do título e do resumo, foram então aplicados os seguintes critérios de inclusão: relevância para abordagem temática, compreendidas no período entre 1999 e 2018 e nos idiomas inglês, português e espanhol. 
O processo seletivo resultou em 50 publicações, sendo 49 artigos e um relatório de consenso (Estratégia Global para Diagnóstico, Condução e Prevenção da DPOC) elaborado e atualizado anualmente pela Iniciativa Global para DPOC (GOLD).

Todas as referências selecionadas foram então organizadas, em ordem cronológica e de citação (se no mesmo ano), no Quadro 1, conforme autor/ano, país de origem do estudo, tipo de estudo, objetivo e critérios de avaliação utilizados na metodologia.

Posteriormente estes estudos foram analisados quanto ao continente de origem e período de publicação nas Figuras 1 e 2, respectivamente.

Quadro 1. Descrição dos estudos selecionados para a revisão; ordem cronológica.

\begin{tabular}{|c|c|c|c|}
\hline Autor/Ano & País & $\begin{array}{l}\text { Tipo de } \\
\text { estudo }\end{array}$ & Objetivo e critérios metodológicos \\
\hline $\begin{array}{l}\text { Scannapieco } \\
\text { FA., } 1999^{9}\end{array}$ & EUA & Revisão & $\begin{array}{l}\text { Descrever as principais doenças respiratórias e propor } \\
\text { mecanismos que explicam o papel de bactérias orais } \\
\text { neste processo. }\end{array}$ \\
\hline $\begin{array}{l}\text { Scannapieco } \\
\text { FA e Ho AW, } \\
2001^{13}\end{array}$ & EUA & $\begin{array}{c}\text { Transversal } \\
\text { retrospectivo* }\end{array}$ & $\begin{array}{l}\text { Avaliar potenciais associações entre doenças } \\
\text { respiratórias e o status de saúde oral através de } \\
\text { informações coletadas de } 13.792 \text { pacientes (com e sem } \\
\text { DPOC) da base de dados NHANES III. }\end{array}$ \\
\hline $\begin{array}{l}\text { Hyman JJ e } \\
\text { Reid BC, } \\
2004^{49}\end{array}$ & EUA & $\begin{array}{c}\text { Estudo } \\
\text { populacional* }\end{array}$ & $\begin{array}{l}\text { Investigar o papel do cigarro na associação entre DP e } \\
\text { DPOC através da comparação de índices periodontais, } \\
\text { espirométricos e história de tabagismo de } 7625 \\
\text { pacientes (com e sem DPOC; fumantes, ex-fumantes e } \\
\text { não fumantes) com dados coletados do NHANES III. }\end{array}$ \\
\hline $\begin{array}{l}\text { Katancik JA et } \\
\text { al., } 2005^{37}\end{array}$ & EUA & Transversal* & $\begin{array}{l}\text { Avaliar a associação entre o estado de saúde } \\
\text { periodontal e a obstrução das vias aéreas através da } \\
\text { comparação de índices periodontais e espirométricos } \\
\text { de } 860 \text { pacientes do Health } A B C \text {. }\end{array}$ \\
\hline $\begin{array}{l}\text { Azarpazhooh } \\
\text { A e Leake JL, } \\
2006^{24}\end{array}$ & Canadá & $\begin{array}{c}\text { Revisão } \\
\text { sistemática* }\end{array}$ & $\begin{array}{l}\text { Investigar evidências para uma possível associação } \\
\text { etiológica entre a saúde oral, a pneumonia e outras } \\
\text { doenças respiratórias. }\end{array}$ \\
\hline $\begin{array}{l}\text { Wang } Z \text { et } \\
\text { al., } 2009^{16}\end{array}$ & China & Caso-controle* & $\begin{array}{l}\text { Avaliar a associação da DP e comportamento } \\
\text { relacionado à saúde oral à DPOC através da } \\
\text { comparação de parâmetros clínicos, periodontais e } \\
\text { questionários em dois grupos (com e sem DPOC). }\end{array}$ \\
\hline $\begin{array}{l}\text { Deo V et al., } \\
2009^{18}\end{array}$ & Índia & $\begin{array}{c}\text { Retrospectivo* } \\
\text { (transversal) }\end{array}$ & $\begin{array}{l}\text { Avaliar a associação entre doenças respiratórias e DP } \\
\text { e correlacionar a severidade da doença periodontal } \\
\text { com a DPOC pela comparação de parâmetros clínicos } \\
\text { e periodontais em dois grupos (com e sem DPOC). }\end{array}$ \\
\hline $\begin{array}{l}\text { Macedo FR et } \\
\text { al., } 2010^{40}\end{array}$ & Brasil & Caso-controle* & $\begin{array}{l}\text { Verificar a associação entre a DP e a doença pulmonar } \\
\text { através da comparação de índices periodontais em } \\
\text { dois grupos (com e sem doença pulmonar). }\end{array}$ \\
\hline $\begin{array}{l}\text { Sharma } \mathrm{N} \text { e } \\
\text { Shamsuddin, } \\
2011^{10}\end{array}$ & Índia & Transversal* & $\begin{array}{l}\text { Comprovar a associaçãa entre doença respiratória e a } \\
\text { DP através da avaliação de parâmetros periodontais } \\
\text { em dois grupos (com e sem doenças respiratórias). }\end{array}$ \\
\hline $\begin{array}{c}\text { Prasanna S, } \\
2011^{19}\end{array}$ & Índia & $\begin{array}{l}\text { Observacional } \\
* \\
\text { (transversal) }\end{array}$ & $\begin{array}{l}\text { Avaliar potencial associação entre a DP e a DPOC e o } \\
\text { status de saúde através da comparação de índices } \\
\text { periodontais em dois grupos (com e sem DPOC). }\end{array}$ \\
\hline $\begin{array}{c}\text { Zhou X et al., } \\
2011^{25}\end{array}$ & China & $\begin{array}{c}\text { Estudo } \\
\text { populacional* }\end{array}$ & $\begin{array}{l}\text { Avaliar a associação da saúde periodontal e } \\
\text { qualidade de vida através de índices periodontais e } \\
\text { aplicação de questionário em } 306 \text { pacientes com } \\
\text { DPOC. }\end{array}$ \\
\hline $\begin{array}{l}\text { Zeng XT et al., } \\
2012^{14}\end{array}$ & China & $\begin{array}{c}\text { Revisão } \\
\text { sistemática } \\
\text { meta-análise* }\end{array}$ & $\begin{array}{l}\text { Avaliar os resultados de } 14 \text { estudos observacionais e } \\
\text { obter uma estimativa mais consistente sobre a } \\
\text { associação da DPOC com a DP. }\end{array}$ \\
\hline
\end{tabular}


Quadro 1. Descrição dos estudos selecionados para a revisão; ordem cronológica. (continua)

\begin{tabular}{|c|c|c|c|}
\hline Autor/Ano & País & $\begin{array}{l}\text { Tipo de } \\
\text { estudo }\end{array}$ & Objetivo e critérios metodológicos \\
\hline $\begin{array}{l}\text { Scott DA e } \\
\text { Krauss JL, } \\
2012^{29}\end{array}$ & EUA & Revisão & $\begin{array}{l}\text { Discutir } \circ \text { papel dos neutrófilos na inflamação } \\
\text { periodontal. }\end{array}$ \\
\hline $\begin{array}{l}\text { Liu } \mathrm{Z} \text { et al., } \\
2012^{41}\end{array}$ & China & Transversal* & $\begin{array}{l}\text { Avaliar a associação entre higiene oral e saúde } \\
\text { periodontal e a frequência de exacerbações da DPOC } \\
\text { através da comparação de parâmetros clínicos } \\
\text { odontológicos e hábitos de higiene oral em dois grupos } \\
\text { (com exacerbações frequentes e raras). }\end{array}$ \\
\hline $\begin{array}{l}\text { Takahashi T et } \\
\text { al., } 2012^{42}\end{array}$ & Japão & $\begin{array}{c}\text { Coorte } \\
\text { prospectivo* }\end{array}$ & $\begin{array}{l}\text { Investigar a associação da titulação do anticorpo lgG } \\
\text { contra } P \text {. gingivalis à frequência de exacerbações da } \\
\text { DPOC através do acompanhamento das exacerbações } \\
\text { de } 93 \text { individuos durante um ano. }\end{array}$ \\
\hline $\begin{array}{l}\text { Bartold PM e } \\
\text { Van Dyke TE, } \\
2013^{6}\end{array}$ & EUA & Revisão* & $\begin{array}{l}\text { Reavaliar conceitos prévios sobre a patogênese da } \\
\text { periodontite, explorando evidências acerca da } \\
\text { influência da microbiota. }\end{array}$ \\
\hline $\begin{array}{l}\text { Usher AKH e } \\
\text { Stockley RA, } \\
2013^{11}\end{array}$ & Inglaterra & Revisão* & $\begin{array}{l}\text { Analisar as evidências epidemiológicas e } \\
\text { fisiopatológicas do processo inflamatório que associa a } \\
\text { DPOC e a DP. }\end{array}$ \\
\hline $\begin{array}{l}\text { Peter KP et } \\
\text { al., } 2013^{12}\end{array}$ & Índia & $\begin{array}{l}\text { Observacional } \\
* \text { (transversal) }\end{array}$ & $\begin{array}{l}\text { Determinar a associação entre DPOC e a DP através } \\
\text { da comparação de parâmetros clínicos e periodontais } \\
\text { em dois grupos (com e sem DPOC). }\end{array}$ \\
\hline $\begin{array}{l}\text { Ledic } \mathrm{K} \text { et al., } \\
2013^{15}\end{array}$ & Croácia & $\begin{array}{l}\text { Caso-controle } \\
\text { retrospectivo* }\end{array}$ & $\begin{array}{l}\text { Avaliar se a DP poderia ser um fator de risco para } \\
\text { DPOC através da comparação de parâmetros } \\
\text { periodontais em dois grupos (com e sem DPOC). }\end{array}$ \\
\hline $\begin{array}{l}\text { Bergstrom J et } \\
\text { al., } 2013^{23}\end{array}$ & Suécia & Transversal & $\begin{array}{l}\text { Descrever a periodontite e o comprometimento da } \\
\text { função pulmonar em fumantes através da avaliação de } \\
\text { parâmetros clínicos, periodontais e questionário em três } \\
\text { grupos (controle, fumantes com e sem DPOC). }\end{array}$ \\
\hline $\begin{array}{l}\text { Quadros AU } \\
\text { et al., } 2013^{36}\end{array}$ & Brasil & Experimental & $\begin{array}{l}\text { Verificar o efeito da nicotina sobre a viabilidade de } \\
\text { LPMN e macrófagos ativados ou não no exsudato } \\
\text { peritoneal de ratos. }\end{array}$ \\
\hline $\begin{array}{l}\text { Barros SP et } \\
\text { al., } 2013^{43}\end{array}$ & EUA & $\begin{array}{c}\text { Coorte } \\
\text { prospectivo* }\end{array}$ & $\begin{array}{l}\text { Avaliar os efeitos do edentulismo, doença periodontal } \\
\text { e biomarcadores inflamatórios sistêmicos na frequência } \\
\text { de eventos adversos em pacientes com DPOC através } \\
\text { da avaliação de } 1635 \text { pacientes (edêntulos e } \\
\text { dentados) do estudo ARIC. }\end{array}$ \\
\hline $\begin{array}{l}\text { Kucukcoskun } M \\
\text { et al., } 2013^{45}\end{array}$ & Turquia & Ensaio clínico* & $\begin{array}{l}\text { Avaliar o efeito do tratamento periodontal sobre a } \\
\text { frequência de exacerbações em dois grupos de } \\
\text { pacientes com DPOC (com e sem tratamento } \\
\text { periodontal) ao longo de um ano. }\end{array}$ \\
\hline $\begin{array}{l}\text { Kassebaum NJ } \\
\text { et al., } 2014^{5}\end{array}$ & EUA & $\begin{array}{l}\text { Revisão } \\
\text { sistemática } \\
\text { meta- } \\
\text { regressão* }\end{array}$ & $\begin{array}{l}\text { Consolidar os dados epidemiológicos sobre a } \\
\text { periodontite severa e gerar estimativas consistentes de } \\
\text { prevalência e incidência para todos os países. }\end{array}$ \\
\hline $\begin{array}{l}\text { Oztekin G et } \\
\text { al., } 2014^{20}\end{array}$ & Turquia & Caso-controle* & $\begin{array}{l}\text { Avaliar os efeitos da DPOC nos tecidos periodontais } \\
\text { através da comparação de índices periodontais e dos } \\
\text { níveis de IL-1 } \beta, \mathrm{PGE}_{2} \text { no fluído crevicular e de PCR } \\
\text { séricos em dois grupos (com e sem DPOC). }\end{array}$ \\
\hline $\begin{array}{l}\text { Ji J et al., } \\
2014^{35}\end{array}$ & Suécia & Transversal* & $\begin{array}{l}\text { Avaliar o processo inflamatório em fumantes nos } \\
\text { diferentes tecidos (boca, vias aéreas e sangue) e a } \\
\text { influência da obstrução das vias aéreas através da } \\
\text { avaliação de biomarcadores inflamatórios em } \\
\text { pacientes saudáveis e fumantes com e sem DPOC. }\end{array}$ \\
\hline $\begin{array}{l}\text { Zhou X et al., } \\
2014^{46}\end{array}$ & China & $\begin{array}{l}\text { Ensaio } \\
\text { controlado } \\
\text { randomizado* }\end{array}$ & $\begin{array}{l}\text { Avaliar o efeito do tratamento periodontal na função } \\
\text { pulmonar e exacerbações em dois grupos de pacientes } \\
\text { com DPOC (com e sem tratamento periodontal), pela } \\
\text { comparação dos índices periodontais e espirométricos e } \\
\text { frequência de exacerbações ao longo de dois anos. }\end{array}$ \\
\hline $\begin{array}{l}\text { Devlin J, } \\
2014^{50}\end{array}$ & Inglaterra & Revisão & $\begin{array}{l}\text { Descrever implicações da DPOC na saúde oral que } \\
\text { devem consideradas pelo cirurgião-dentista. }\end{array}$ \\
\hline
\end{tabular}


Quadro 1. Descrição dos estudos selecionados para a revisão; ordem cronológica. (continuação)

\begin{tabular}{|c|c|c|c|}
\hline Autor/Ano & País & $\begin{array}{l}\text { Tipo de } \\
\text { estudo }\end{array}$ & Objetivo e critérios metodológicos \\
\hline $\begin{array}{l}\text { Putcha } \mathrm{N} \text { et } \\
\text { al., } 2015^{3}\end{array}$ & EUA & Revisão* & $\begin{array}{l}\text { Revisar o conhecimento atual em torno das } \\
\text { comorbidades da DPOC. }\end{array}$ \\
\hline $\begin{array}{l}\text { Shen TC et al., } \\
2015^{21}\end{array}$ & China & Coorte* & $\begin{array}{l}\text { Avaliar se a DPOC é um fator de risco para DP através } \\
\text { de informações coletadas de } 66.094 \text { pacientes (com e } \\
\text { sem DPOC) da base de dados NHRI. }\end{array}$ \\
\hline $\begin{array}{l}\text { Bhavsar NV et } \\
\text { al., } 2015^{39}\end{array}$ & Índia & Transversal & $\begin{array}{l}\text { Avaliar a associação entre a DP e comportamento com } \\
\text { a saúde oral e a DPOC através da comparação dos } \\
\text { indices periodontais e dos niveis séricos e salivares de } \\
\text { PCR entre dois grupos (com e sem DPOC). }\end{array}$ \\
\hline $\begin{array}{l}\text { Chung JH et } \\
\text { al., } 2016^{22}\end{array}$ & $\begin{array}{l}\text { Coreia do } \\
\text { Sul }\end{array}$ & Transversal* & $\begin{array}{l}\text { Avaliar se a higiene oral e o autocuidado com a saúde } \\
\text { periodontal estão associados à DPOC, através de } \\
\text { informações coletadas de } 5.878 \text { pacientes (com e sem } \\
\text { DPOC) da base de dados NHANES coreana. }\end{array}$ \\
\hline $\begin{array}{l}\text { Martinez FD, } \\
2016^{27}\end{array}$ & EUA & Revisão* & $\begin{array}{l}\text { Discutir as questões etiológicas envolvidas no } \\
\text { desenvolvimento da DPOC ao longo da vida. }\end{array}$ \\
\hline $\begin{array}{c}\text { Barnes PJ, } \\
2016^{28}\end{array}$ & Inglaterra & Revisão* & $\begin{array}{l}\text { Discutir a natureza da inflamação em pacientes com } \\
\text { DPOC e os mecanismos moleculares que possam } \\
\text { propiciar o surgimento de terapias mais efetivas. }\end{array}$ \\
\hline $\begin{array}{l}\text { Madalli } R \text { et } \\
\text { al., } 2016^{34}\end{array}$ & Índia & $\begin{array}{l}\text { Coorte } \\
\text { prospectivo }\end{array}$ & $\begin{array}{l}\text { Avaliar o patógeno } P \text {. gingivalis no escarro de } 30 \\
\text { pacientes com DPOC e periodontite antes e seis meses } \\
\text { após o tratamento periodontal e correlacioná-lo com } \\
\text { parâmetros respiratórios e periodontais. }\end{array}$ \\
\hline $\begin{array}{l}\text { Shen TC et al., } \\
2016^{47}\end{array}$ & China & $\begin{array}{l}\text { Coorte } \\
\text { retrospectivo } \\
\text { de base } \\
\text { populacional* }\end{array}$ & $\begin{array}{l}\text { Investigar se o tratamento periodontal reduz o risco de } \\
\text { eventos adversos em pacientes com DPOC através da } \\
\text { avaliação de } 11.124 \text { pacientes com dados coletados } \\
\text { do NHRI de Taiwan por } 5 \text { anos. }\end{array}$ \\
\hline $\begin{array}{l}\text { Henke } C \text { et } \\
\text { al., } 2016^{48}\end{array}$ & Alemanha & $\begin{array}{l}\text { Transversal } \\
\text { prospectivo* }\end{array}$ & $\begin{array}{l}\text { Investigar a possivel associaçãa entre a função } \\
\text { pulmonar e a saúde oral através da comparação de } \\
\text { parâmetros clínicos, odontológicos e espirométricos de } \\
206 \text { pacientes. }\end{array}$ \\
\hline $\begin{array}{l}\text { Balta MG et } \\
\text { al., } 2017^{1}\end{array}$ & Holanda & Revisão* & $\begin{array}{l}\text { Apresentar informações recentes sobre a resolução da } \\
\text { inflamação periodontal. }\end{array}$ \\
\hline $\begin{array}{l}\text { Hobbins S et } \\
\text { al., } 2017^{4}\end{array}$ & Inglaterra & Revisão* & $\begin{array}{l}\text { Analisar as evidências que sustentam a hipótese de que } \\
\text { a DPOC e a DP estão associadas. }\end{array}$ \\
\hline $\begin{array}{l}\text { Holmstrup P et } \\
\text { al., } 2017^{8}\end{array}$ & Dinamarca & Revisão* & $\begin{array}{l}\text { Apresentar evidências da associação da periodontite } \\
\text { com doenças sistêmicas. }\end{array}$ \\
\hline $\begin{array}{l}\text { Nakayama M } \\
\text { e Ohara N, } \\
2017^{30}\end{array}$ & Japão & Revisão* & $\begin{array}{l}\text { Discutir a nova caracterização molecular e biológica } \\
\text { dos fatores de virulência da } P \text {. gingivalis. }\end{array}$ \\
\hline $\begin{array}{l}\text { Moeintaghavi } \\
\text { A et al., } \\
2017^{32}\end{array}$ & Irã & Transversal & $\begin{array}{l}\text { Comparar a expressão do gene IL-1 } \beta \text { e IL- } 12 \text { no tecido } \\
\text { gengival de fumantes e não fumantes, com e sem } \\
\text { doença periodontal. }\end{array}$ \\
\hline $\begin{array}{l}\text { Bosshardt DD, } \\
2017^{33}\end{array}$ & Suiça & Revisão & $\begin{array}{l}\text { Descrever a patogênese, histopatologia e } \\
\text { consequências da bolsa periodontal. }\end{array}$ \\
\hline $\begin{array}{l}\text { Terashima T et } \\
\text { al., } 2017^{44}\end{array}$ & Japão & Caso-controle* & $\begin{array}{l}\text { Avaliar a hipótese de que pacientes DPOC têm saúde } \\
\text { periodontal mais precária e menor status nutricional } \\
\text { através da avaliação de indices periodontais, IMC e } \\
\text { albumina sérica em dois grupos (com e sem DPOC). }\end{array}$ \\
\hline GOLD, $2018^{2}$ & Mundial & $\begin{array}{l}\text { Relatório de } \\
\text { consenso* }\end{array}$ & $\begin{array}{l}\text { Estratégia global para o diagnóstico, condução e } \\
\text { prevenção da DPOC. }\end{array}$ \\
\hline $\begin{array}{l}\text { Baek K et al., } \\
2018^{7}\end{array}$ & $\begin{array}{l}\text { Coreia do } \\
\text { Sul }\end{array}$ & Transversal & $\begin{array}{l}\text { Comparar a microbiota do tecido gengival com a } \\
\text { microbiota obtida do biofilme de lesões periodontais } \\
\text { através da análise do gene } 16 \mathrm{~S} \text { rRNA e carga } \\
\text { bacteriana em } 7 \text { pacientes. }\end{array}$ \\
\hline $\begin{array}{l}\text { Bhatt SP et al., } \\
2018^{26}\end{array}$ & EUA & $\begin{array}{c}\text { Coorte } \\
\text { populacional } *\end{array}$ & $\begin{array}{l}\text { Comparar os índices "duração do tabagismo" versus } \\
\text { "pacote-anos" como métodos de estimativa para o } \\
\text { risco de DPOC através da análise de dados de } 10.187 \\
\text { pacientes (COPDGene) fumantes e ex-fumantes. }\end{array}$ \\
\hline
\end{tabular}


Quadro 1. Descrição dos estudos selecionados para a revisão; ordem cronológica. (conclusão)

\begin{tabular}{|c|c|c|l|}
\hline Autor/Ano & País & $\begin{array}{c}\text { Tipo de } \\
\text { estudo }\end{array}$ & \multicolumn{1}{c|}{ Objetivo e critérios metodológicos } \\
\hline $\begin{array}{c}\text { Shibata K, } \\
2018^{31}\end{array}$ & Japão & Revisão* & $\begin{array}{l}\text { Abordar os aspectos históricos do papel patológico dos } \\
\text { inflamassomas nas doenças periodontais bem como da } \\
\text { sua ativação pelos } \text { P. gingivalis para o desenvolvimento } \\
\text { de estratégias terapêuticas futuras. }\end{array}$ \\
\hline $\begin{array}{c}\text { Barrionuevo } \\
\text { AMP et al., } \\
2018^{38}\end{array}$ & Noruega & Populacional* & $\begin{array}{l}\text { Avaliar se a saúde periodontal está relacionada à } \\
\text { função pulmonar através da comparação de índice } \\
\text { periodontal e espirometria em 656 participantes do } \\
\text { RHINESSA e ECRHS. }\end{array}$ \\
\hline
\end{tabular}

* Conforme definição dos autores.

et al: e outros; GOLD: Global Initiative for Chronic Obstructive Lung Disease; DPOC: doença pulmonar obstrutiva crônica; EUA: Estados Unidos da América; DP: doença periodontal; NHANES III: National Health Nutrition Examination Survey III; IL-1 $\beta$ : interleucina 1 $\beta$, PGE2: prostaglandina E2; PCR: proteína C reativa; NHRI: National Health Research Institutes; COPDGene: multicentro Chronic Obstructive Pulmonary Disease Gene; P. gingivalis: Porphyromonas gingivalis; LPMN: leucócitos polimorfonucleares; Health ABC: Health, Aging and Body Composition; RHINESSA: Respiratory Health in Northern Europe, Spain and Australia; ECRHS: European Community Respiratory Health Survey; IgG: imunoglobulina G; ARIC: Atherosclerosis Risk in Communities; IMC: índice de massa corpórea.

Figura 1. Distribuição dos estudos por continente de origem.

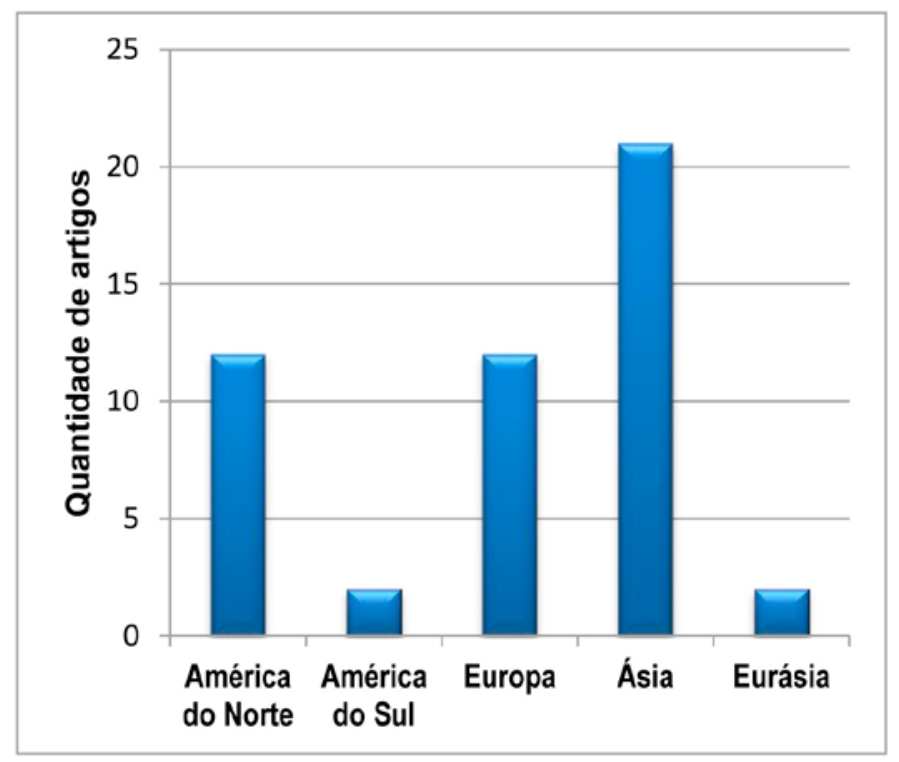

Figura 2. Distribuição dos estudos por período de publicação.

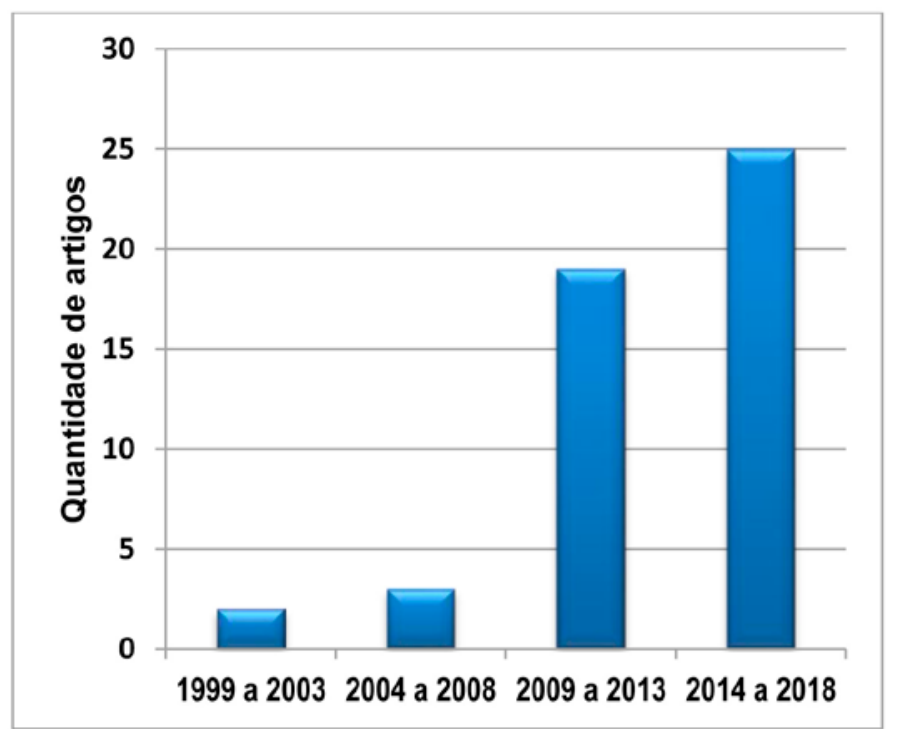




\section{Revisão da literatura}

A seguinte revisão aborda a associação entre a DPOC e a doença periodontal partindo da caracterização de cada uma das doenças e da fisiopatologia da associação entre elas, descrição da doença periodontal nos pacientes com DPOC, implicações da doença periodontal no curso da DPOC e os fatores confundidores dessa associação. Serão discutidas as evidências científicas voltadas para a maior abrangência e eficácia no tratamento da DPOC, a fim de promover um atendimento multidisciplinar e integral.

\section{A Doença Pulmonar Obstrutiva Crônica (DPOC)}

A DPOC é caracterizada por uma limitação crônica e recorrente ao fluxo de ar, causada por alterações nas vias aéreas e/ou alveolares que geralmente são provocadas pela exposição a partículas ou gases nocivos. A inflamação crônica resulta em alterações estruturais, estreitamento das pequenas vias aéreas, destruição do parênquima pulmonar e perda da elasticidade. $O$ enfisema (destruição das superfícies de trocas gasosas nos pulmões) e a bronquite crônica representam anormalidades estruturais presentes nos pacientes com DPOC ${ }^{2}$.

Em 1998, com o objetivo de ampliar o conhecimento da DPOC, um grupo de cientistas incentivou o US National Heart, Lung, and Blood Institute e a Organização Mundial da Saúde a formarem o Global Initiative for Chronic Obstructive Lung Disease (GOLD). Em 2001 foi escrito o primeiro relatório de consenso Global Strategy for the Diagnosis, Management, and Prevention of COPD que, desde então, vem sendo anualmente atualizado. Ao longo destes anos, a prevalência, morbidade e mortalidade da DPOC vêm aumentando significativamente. De acordo com - relatório de 2006, nos Estados Unidos, a DPOC era a quarta principal causa de morbidade e mortalidade, com projeção para estar em quinto lugar em 2020. No relatório mais recente, de 2018, a DPOC já é a quarta causa de morte no mundo, com perspectiva de ocupar o terceiro lugar em 20202. No Brasil, a DPOC está entre as poucas causas de morte que apresentam crescimento, sendo, a terceira causa de morte entre as doenças crônicas não transmissíveis.
A DPOC tem como principal fator de risco o tabagismo. Estudo realizado em $2018^{26}$ demonstrou que a duração do tabagismo proporciona estimativas de risco mais consistente do que a quantidade de cigarros fumados por dia e o índice composto por "anos-maço". Neste contexto, deve ainda ser considerada como fator de risco, a exposição a outros gases e partículas nocivos - em países em desenvolvimento, a polvição proveniente do fogão à lenha está associada ao risco elevado de desenvolver a DPOC.

Além da exposição à fumaça, há também outros fatores de risco cuja presença e/ou interação podem promover ou favorecer o desenvolvimento da DPOC, dentre os quais estão: fatores genéticos (como a deficiência da alfa- 1 antitripsina), idade (relacionada a um efeito cumulativo da exposição), gênero (associado também ao hábito do tabagismo), crescimento e desenvolvimento do pulmão (desde a gestação até adolescência), histórico de infecções respiratórias na infância e status socioeconômico ${ }^{2,26,27}$.

A fisiopatologia da DPOC está relacionada ao aumento da quantidade de células inflamatórias, principalmente neutrófilos, macrófagos e linfócitos $T$ e $B$ e também à ativação de células estruturais, como as células epiteliais das vias aéreas e alveolares, células endoteliais e fibroblastos. Estas células produzem mediadores que estão diretamente envolvidos na modificação e ampliação da reposta inflamatória normal nos pacientes com DPOC, como prostaglandinas (PG), leucotrienos (LT), fator de necrose tumoral (TNF), inflamassomas (que regulam a expressão das interleucinas (IL) $1 \beta$ e 18), quimiocinas, proteases, como as metaloproteinases (MMP), e proteína C-reativa $(P C R)^{2,20,28}$. A presença, na corrente sanguínea, de mediadores inflamatórios similares aos que são encontrados no pulmão de pacientes com DPOC pode estar relacionada às suas manifestações sistêmicas, podendo agravar as suas doenças comórbidas $^{3,28}$.

Dentre as diversas comorbidades associadas à DPOC, estão as doenças cardiovasculares, osteoporose, síndrome metabólica e diabetes, refluxo gastroesofágico, ansiedade e depressão ${ }^{2,3,4}$. Recentemente tem havido um maior interesse na associação da DPOC com a doença periodontal, embora os mecanismos desta associação ainda não estejam completamente elucidados ${ }^{4}$. 
A DPOC é uma doença prevenível e tratável, mas que ainda representa uma parcela substancial dos gastos com saúde. A ampliação do conhecimento acerca da sua fisiopatologia e interações sistêmicas possibilita um aperfeiçoamento da prevenção e do tratamento com consequente redução da morbidade e mortalidade decorrentes da doença.

\section{A Doença Periodontal}

A doença periodontal atinge, em diferentes níveis de severidade, mais da metade da população mundial, estando, a periodontite severa, entre as dez condições mais prevalentes do mundo ${ }^{5}$.

A patogênese da doença periodontal é desencadeada pela interrupção, mediada pelo pacientehospedeiro, da homeostase microbiana presente no biofilme ${ }^{1,6}$. O consequente processo inflamatório crônico pode provocar inflamação gengival com infiltrado de células inflamatórias e levar a destruição dos tecidos de suporte e inserção periodontal e do osso alveolar, podendo, inclusive, culminar na perda da unidade dentária ${ }^{1,6,7}$.

A doença periodontal é caracterizada por um infiltrado neutrofílico. Encontrados em grande quantidade no sulco gengival, os neutrófilos são considerados a principal célula protetora dos tecidos periodontais devido à sinergia entre o seu efeito fagocítico e também secretor de citocinas pró-inflamatórias e de espécies reativas de oxigênio (ERO). Entretanto, a perda desta homeostase imunológica permite estabelecer uma associação entre a infiltração de neutrófilos nos tecidos periodontais e a gravidade e progressão da doença periodontal ${ }^{1,29}$.

Além da microbiota presente no biofilme, estudo publicado em 2018 demonstrou que há ainda várias "comunidades" bacterianas muito complexas dentro do tecido gengival de lesões periodontais que podem, potencialmente, funcionar como um reservatório para infecção persistente. A grande quantidade de Porphyromonas gingivalis ( $P$. gingivalis) e Fusobacterium nucleatum (F.nucleatum) nas comunidades de tecidos sugere que essas duas espécies também interajam para facilitar a sobrevivência no novo ambiente $^{7}$.
A P.gingivalis é o principal patógeno da doença periodontal, possuindo, dentre outros fatores de virulência e patogenicidade, o receptor de hemoglobina $(\mathrm{HbR})$, que induz a expressão da IL-8 em células epiteliais gengivais, e as proteases gingipains, que tem forte atividade proteolítica ${ }^{30}$.

Em 2002, os inflamassomas foram descritos pela primeira vez como um complexo de proteínas envolvido no processamento da pró-IL- $1 \beta$ para a sua forma ativa IL-1 $\beta$. Apenas em 2009, a sua ligação com a doença periodontal foi descrita, demonstrando-se que a P.gingivalis, além de outras bactérias, pode estar envolvida na ativação dos inflamassomas, embora ainda hoje os relatos na literatura sejam controversos acerca do mecanismo desta ativação ${ }^{31}$. A IL-1 $\beta$ desempenha um papel chave na doença periodontal, induzindo a produção de mediadores inflamatórios, formação de osteoclastos, expressão de MMP e a morte de células produtoras de matriz, resultando na destruição do osso alveolar e tecidos periodontais $^{31,32}$. Este quadro de destruição óssea pela reabsorção osteoclástica, inflamação, destruição tecidual e desprendimento do epitélio juncional caracteriza a bolsa periodontal ${ }^{33}$.

No periodonto, os mecanismos normais de defesa contra micro-organismos e seus produtos são assegurados pela alta permeabilidade da superfície livre do epitélio juncional, que permite que fluidos sulculares e células inflamatórias e imunes, principalmente neutrófilos, atinjam o fundo do sulco e entrem na cavidade oral. Entretanto, este sistema aberto, que não possui uma barreira física na forma de uma camada celular queratinizada, pode permitir que microrganismos e seus produtos invadam o epitélio juncional. A destruição dessa integridade estrutural leva, consequentemente, a formação da bolsa, desequilibrando este delicado sistema de defesa. As células de defesa e as macromoléculas são diretamente descarregadas na bolsa periodontal e a maioria das células epiteliais diretamente no biofilme. $O$ adelgaçamento do epitélio e sua ulceração aumentam a chance de invasão de micro-organismos e seus produtos no tecido conjuntivo. Instala-se então um círculo vicioso, rompido apenas com intervenção terapêutica ${ }^{33}$.

Assim como para a DPOC, o tabagismo também é um fator de risco modificador para a doença perio- 
dontal. Foi demonstrado ${ }^{32}$ que em pacientes fumantes com periodontite, o nível de expressão do gene da IL-1 $\beta$ foi significativamente menor do que nos não fumantes, o que estaria relacionado à menor intensidade dos sintomais inflamatórios na gengiva de pacientes tabagistas. No entanto, os autores destacam que, apesar de sinais inflamatórios mais brandos, o grau de destruição periodontal e perda óssea nesses pacientes seriam maiores do que os pacientes que não fumam.

Nos últimos anos, tem havido um crescente interesse científico na associação da doença periodontal com outras afecções sistêmicas, muitas das quais também com alta prevalência na população geral, como por exemplo, doenças cardiovasculares, diabetes tipo 2, artrite reumatóide, osteoporose e doenças respiratórias. Embora as vias inflamatórias compartilhadas fundamentem esta associação, uma relação direta de causalidade será difícil de ser alcançada por questões éticas. Algumas dessas comorbidades podem melhorar por tratamento periodontal, podendo existir uma relação bidirecional entre elas e a doença periodontal8. Neste contexto, a associação entre a doença periodontal e a DPOC tem sido foco de profundo interesse.

\section{Fisiopatologia da associação Doença Periodontal - DPOC}

Em todos os estudos que se objetiva avaliar a associação entre a doença periodontal e a DPOC é de fundamental importância que sejam considerados os diversos mecanismos moleculares que podem fundamentar esta associação. Muitas pesquisas sugerem que esta ligação acontece através da presença de bactérias patogênicas na cavidade oral, aspiradas ou através de acesso à corrente sanguínea ${ }^{4,9,10,33}$, e também pelo fato de ambas serem doenças inflamatórias mediadas por neutrófilos ${ }^{4,11,28,33}$.

O biofilme pode funcionar como um meio para colonização de bactérias respiratórias, que representaria um reservatório destes patógenos, podendo culminar em infecções respiratórias ${ }^{9,34}$. Os mecanismos potenciais de ação das bactérias orais na patogênese da infecção respiratória são: aspiração de bactérias orais diretamente para os pulmões; alterações na superfície mucosa provocada por mediadores da inflamação periodontal, promovendo a adesão e colonização por patógenos respiratórios; destruição da película protetora com redução da capacidade de eliminação de patógenos; e alterações no epitélio respiratório promovidas por citocinas originadas dos tecidos periodontais?

Tanto a doença periodontal como a DPOC são condições inflamatórias caracterizadas pela perda de tecido conjuntivo local. Embora a patologia destas duas doenças seja complexa e envolva muitos tipos de células e mediadores inflamatórios, as duas condições são sustentadas por processos fisiopatológicos semelhantes, predominantemente caracterizadas por inflamação neutrofílica ${ }^{4,11,28,33}$. Neste sentido, alguns autore ${ }^{20}$ demonstraram maiores níveis de PCR, IL-1 $\beta$ e PG-E2 no fluído crevicular gengival e séricos de PCR em pacientes do grupo DPOC. Adicionalmente, foi também demonstrada ${ }^{35}$ uma forte correlação entre os níveis salivares de IL-8 e MMP9 e a severidade da inflamação periodontal, estabelecendo, ao mesmo tempo, uma relação negativa com função pulmonar. Estes achados sugerem que estes marcadores inflamatórios estão relacionados com a severidade da DPOC.

Na gênese desta associação, a nicotina, principal componente farmacológico do tabaco, reduz a citotoxicidade e favorece a sobrevida dos leucócitos polimorfonucleares (LPMN) não-ativados, em concentrações próximas àquelas encontradas na cavidade oral e no fluido broncoalveolar de fumantes. Este efeito citoprotetor da nicotina sobre o sistema de macrófago e, principalmente, sobre os LPMN, aumentaria a sua viabilidade, podendo favorecer exacerbações pela maior produção de $\mathrm{ERO}^{36}$.

\section{A doença periodontal nos pacientes com DPOC}

Em grande parte das pesquisas clíni$\operatorname{cas}^{10,12,13,15,17-19,20-22,37-40}$, a avaliação da doença periodontal é realizada principalmente através dos seus índices e parâmetros clínicos: índice de placa (IP), índice de sangramento gengival (IG), profundidade de bolsa à sondagem (PS) e nível de inserção clínica (NIC).

Na maioria dos estudos utilizados nesta revisão, os parâmetros periodontais apresentaram-se mais graves nos pacientes do grupo DPOC, sugerindo a doença periodontal como um fator de risco para 
a DPOC. Alguns estudos populacionais foram desenvolvidos com a finalidade de avaliar indicadores do status da saúde oral em pacientes com DPOC $^{13,22,37,38}$. Os resultados destes estudos demonstraram que pacientes com DPOC tiveram maior perda de inserção periodontal e que esta perda também estava relacionada com maior redução da função pulmonar e maior risco de desenvolver DPOC. Estes dados foram também corroborados em estudos observacionais ${ }^{10,12,15,17-19,39}$, com exceção apenas de um estudo ${ }^{40}$, cujo "grupo caso" era formado por $40 \%$ de pacientes com DPOC, sendo os demais com pneumonia adquirida. Neste estudo houve diferença estatística apenas no IP.

Em outra direção, sob uma análise menos pesquisada, alguns estudos demonstraram que a DPOC, tanto pelos seus efeitos sistêmicos como pelo seu tratamento, exerce um efeito prejudicial no desenvolvimento da doença periodontal ${ }^{20,21}$. A correlação entre os parâmetros clínicos periodontais, como índice de placa, número de dentes e índice de sangramento, e os mediadores inflamatórios presentes no fluido crevicular e na corrente sanguínea sugere que a DPOC pode ser um fator de risco para a doença periodontal ${ }^{20}$.

Embora muitos destes estudos não permitam inferir uma relação de causalidade entre a DPOC e a doença periodontal, fornecem importantes evidências para fundamentar pesquisas futuras ${ }^{14}$.

\section{Implicações da doença periodontal no curso da DPOC}

Em face destes resultados anteriormente referenciados, na literatura há também estudos que avaliaram a relação entre eventos respiratórios adversos nos pacientes com DPOC e a sua saúde periodontal $^{25,41-44}$ bem como em resposta ao tratamento periodontal ${ }^{24,34,45-47}$, sugerindo que a promoção de uma melhor saúde periodontal deve ser uma estratégia preventiva a ser implementada no tratamento da DPOC.

Uma das maiores complicações da DPOC é a ocorrência de exacerbações, que se caracterizam pela piora do quadro clínico, sendo a principal causa de hospitalização e mortalidade nestes pacientes. A maior frequência de exacerbações associada a um precário quadro de saúde periodontal já foi demonstrada na literatura, correlacionando-se, inclusive, com menor número de dentes, maior índice de placa e baixa frequência de escovação ${ }^{41}$.

Acerca dos aspectos imunológicos desta implicação, apesar de a maior frequência de exacerbações não ter sido associada a maiores valores de anticorpos IgG contra P.gingivalis, os autores ${ }^{42}$ ressaltaram que este achado se opôs a sua hipótese inicial e concluem que este anticorpo pode ser um preditor independente da frequência de exacerbações. Em uma análise mais ampla, outro estudo ${ }^{43}$ correlacionou os mais altos níveis séricos de IL-6 e PCR ao maior risco de exacerbações no curso da DPOC, associando-se ainda à condição periodontal mais precária e edentulismo.

Em investigação acerca da associação entre os parâmetros periodontais e o estado nutricional em pacientes com DPOC, foi demonstrado que o menor número de dentes, maior índice de sangramento e maior profundidade de bolsa associaram-se ao menor nível de albumina sérica. Os resultados sugerem que a associação entre a condição periodontal precária e a hipoalbuminemia favorece a instalação de um quadro inflamatório em pacientes com DPOC ${ }^{44}$.

$\mathrm{Na}$ literatura, os resultados de muitos estudos sugerem que a instituição do tratamento periodontal pode melhorar o curso da DPOC ${ }^{24,34,45-47}$. O tratamento periodontal reduz a contagem de $P$. gingivalis e está associado a uma melhora da função pulmonar (volume expiratório forçado no primeiro segundol/capacidade vital forçada) ${ }^{34}$. Em estudos prospectivos, foi constatado que a instituição do tratamento periodontal durante 0 período de $\mathrm{um}^{45} \mathrm{e}$ dois 46 anos em pacientes com DPOC implicou em redução na frequência de exacerbações, ao contrário do grupo que não recebeu tratamento, no qual pôde ser constatado um aumento. Estes resultados são corroborados por estudo populacional ${ }^{47} \mathrm{com}$ 11.124 pacientes identificados com DPOC entre 2000 e 2006 (dados coletados do National Health Research Institutes (NHRI), Taiwan).

Considerando ainda a significativa redução na qualidade de vida demonstrada em alguns estudos ${ }^{23,25}$, a instituição do tratamento periodontal e promoção de saúde bucal para estes pacientes implicariam em economias significativas de custo e melhoria da qualidade de vida com benefícios em âmbito odontológico, sistêmico e também psicológico ${ }^{14,17,24,25,39}$. 


\section{Fatores confundidores na associação Doença Periodontal - DPOC}

Apesar de muitos estudos investigarem os mecanismos de associação entre a doença periodontal e a DPOC, alguns autores analisam que muitos destes estudos não consideram que estas duas patologias compartilham muitos fatores de risco em comum, como idade, tabagismo, sexo, doenças sistêmicas, status socioeconômico e nutricional, nível de escolaridade, uso de medicações e aspectos comportamentais relacionados ao autocuidado, incluindo a saúde oral e interesse e condições de acesso aos serviços de saúde. Para esta análise, é de fundamental importância considerar a influência direta destes, que funcionariam como fatores confundidores, na associação entre a doença periodontal e a DPOC ${ }^{4}$. No caso da doença periodontal, a própria resposta do hospedeiro é modulada por fatores genéticos, respostas imunológicas e inflamatórias, estresse, tabagismo, dieta, determinantes sociais e status de sua saúde geral ${ }^{6}$.

Uma das limitações dos estudos que investigam esta associação é a impossibilidade de se diferenciar completamente o impacto do tabagismo (enquanto fator de risco atual ou prévio) associado à presença da DPOC na condição periodontal ${ }^{23,26,27,32,41,48,49}$. Em estudo ${ }^{48}$ para avaliar a associação entre vários parâmetros de saúde oral, incluindo periodontais, e as medidas espirométricas em 206 pacientes sem DPOC, os autores ressaltam que a análise estatística das associações foi fortemente influenciada por outros cofatores como o tabagismo e a idade. Semelhante influência foi encontrada em estudo ${ }^{23}$ que avaliou a saúde periodontal em pacientes não fumantes e fumantes com e sem DPOC. Apesar de alguns índices periodontais terem sido mais graves nos pacientes fumantes (sem diferença pela presença da DPOC), os autores ressaltam que quando a covariável idade foi controlada no modelo multivariado, a associação entre dados espirométricos e as medidas do status periodontal não se manteve.

Há ainda que se considerar a influência do uso de medicações sobre a associação DPOC-doença periodontal. Medicações utilizadas no tratamento da DPOC, como beta-agonistas, corticoides, antagonistas muscarínicos e antibióticos, podem ter profun- das implicações na cavidade oral, como xerostomia e candidíase ${ }^{50}$. Pacientes com DPOC tratados com corticoides (inalado ou sistêmico) tiveram um risco maior de desenvolver doença periodontal possivelmente pelo seu efeito na redução da densidade óssea e imunossupressor ${ }^{21}$. Paradoxalmente, foi demonstrado em um estudo ${ }^{41}$ que os índices periodontais foram melhores nos pacientes com DPOC que tiveram maior frequência de exacerbações. Os autores especulam que este fenômeno pode estar relacionado ao uso frequente de antibiótico durante as exacerbações, que teriam um efeito protetor inibitório sobre a doença periodontal.

Quanto aos aspectos comportamentais, além dos índices periodontais, a frequência de escovação, uso do fio dental, visitas ao dentista ${ }^{16}$ e edentulismo ${ }^{43}$ foram relacionados ao maior risco de desenvolver DPOC. Segundo os autores, estes dados estão relacionados ao conhecimento, atitudes e crenças relacionadas à saúde que, certamente, também influenciam o risco e/ou o curso da DPOC. Os autores destacam, portanto, a importância não só do tratamento periodontal, mas também da orientação para o autocuidado adequado e regular que estes pacientes devem receber.

Somando-se ainda a este contexto, faz-se importante destacar que a maioria dos estudos discutidos aqui nesta revisão pertence ao continente asiático e europeu. Além dos fatores confundidores já mencionados, deve-se também considerar o perfil populacional socioeconômico e cultural característico de cada região que podem ainda corroborar com esta análise.

\section{Considerações finais}

A DPOC é atualmente a quarta causa de morte no mundo, com perspectiva de estar em terceiro lugar já em 2020. Embora haja uma quantidade crescente de publicações acerca da sua associação com a doença periodontal, o pequeno número de estudos desenvolvidos na América do Sul destaca a necessidade de mais investigações científicas nesta região.

A análise dos estudos incluídos nesta revisão fornece evidências da potencial associação da doença periodontal com a DPOC, ambas caracterizadas por 
um mecanismo fisiopatológico essencialmente inflamatório. Estudos sugerem que assim como a doença periodontal pode afetar o desenvolvimento e/ ou curso da DPOC, este mecanismo pode acontecer também numa relação inversa, em que a DPOC pode também funcionar como um fator de risco para a doença periodontal. A existência de muitos fatores de risco e/ou confundidores para ambas as patologias deve ser considerada na análise de evidências científicas e clínicas.

Esta revisão apresenta, portanto, um embasamento científico para a implantação de estratégias futuras que propiciem um aumento da abrangência e aprimoramento da resolutividade no tratamento da DPOC. A complementação do protocolo de acompanhamento e tratamento de pacientes com DPOC, através da introdução do tratamento periodontal desde a atenção básica e da implementação de ações educativas na área de prevenção e promoção de saúde bucal, promoverá um atendimento multidisciplinar e integrado e, consequentemente, de maior efetividade.

\section{Contribuição dos autores}

Libório-Lago CC contribuiu na elaboração da ideia inicial, concepção e desenho do estudo, busca bibliográfica, análise e interpretação dos dados, redação do artigo e revisão das sucessivas versões. Camelier FWR contribuiu na elaboração da ideia inicial, na análise e interpretação dos dados e revisão das sucessivas versões. Santos CO contribuiu na concepção e desenho do estudo, busca bibliográfica, redação do artigo e revisão das sucessivas versões. Coutinho MR contribuiu na elaboração da ideia inicial, análise dos dados e aprovação da versão final do artigo. Bittencourt $S$ contribuiu na concepção e desenho do estudo e aprovação da versão final do artigo. Camelier AA contribuiu na elaboração da ideia inicial, concepção e desenho do estudo, redação do artigo e revisão das sucessivas versões.

\section{Conflito de interesses}

Os autores declaram não haver nenhum conflito de interesses direta ou indiretamente relacionado ao conteúdo do manuscrito.

\section{Referências}

1. Balta MG, Loos BG, Nicu EA. Emerging concepts in the resolution of periodontal inflammation: a role for resolvin E1. Front Immunol. 2017;8:1682. doi: 10.3389/ fimmu.2017.01682
2. Global Initiative for Chronic Obstructive Lung Disease. Global Strategy for the Diagnosis, Management, and Prevention of Chronic Obstructive Pulmonary Disease [Internet]. 2018 (acesso em 2018 fev. 22). Disponível em: http://goldcopd.org/wp-content/uploads/2017/1 1/GOLD2018-v6.0-FINAL-revised-20-Nov_WMS.pdf

3. Putcha N, Drummond MB, Wise RA, Hansel NN. Comorbidities and chronic obstructive pulmonary disease: prevalence, influence on outcomes, and management. Semin Respir Crit Care Med. 2015;36(4):575-91. doi: 10.1055/s$\underline{0035-1556063}$

4. Hobbins S, Chapple ILC, Sapey E, Stockley RA. Is periodontitis a comorbidity of COPD or can associations be explained by shared risk factors/behaviors? Int J Chron Obstruct Pulmon Dis. 2017;1 2:1339-49. doi: 10.2147/ COPD.S127802

5. Kassebaum NJ, Bernabé E, Dahiya M, Bhandari B, Murray CJL, Marcenes W. Global burden of severe periodontitis in 1990-2010: a systematic review and Meta-regression. J Dental Res. 2014;93(1 1):1045-53. doi: $\underline{10.1177 / 0022034514552491}$

6. Bartold PM, Van Dyke TE. Periodontitis: a host-mediated disruption of microbial homeostasis. Unlearning learned concepts. Periodontol 2000. 2013;62(1):203-17. doi: $10.1111 / \mathrm{i} .1600-0757.2012 .00450 . x$

7. Baek K, Ji S, Choi Y. Complex intratissue microbiota forms biofilms in periodontal lesions. J Dent Res. 2018;97(2):192200. doi: $10.1177 / 0022034517732754$

8. Holmstrup P, Damgaard C, Olsen I, Klinge B, Flyvbjerg A, Nielsen $\mathrm{CH}$ et al. Comorbidity of periodontal disease: two sides of the same coin? An introduction for the clinician. J Oral Microbiol. 2017;9(1):1332710. doi: 10.1080/20002297.2017.1332710

9. Scannapieco FA. Role of oral bacteria in respiratory infection. J Periodontol. 1999;70(7):793-802. doi: 10.1902/ jop. 1999.70.7.793

10. Sharma N, Shamsuddin H. Association between respiratory disease in hospitalized patients and periodontal disease: a cross-sectional study. J Periodontol. 2011 ;82(8):1 155-60. doi: 10.1902/iop.2011.100582

11. Usher AKH, Stockley RA. The link between chronic periodontitis and COPD: a common role for the neutrophil. BMC Med. 2013;1 1:241. doi: 10.1 186/1741-7015-11-241

12. Peter KP, Mute BR, Doiphode SS, Bardapurkar SJ, Borkar MS, Raje DV. Association between periodontal disease and chronic obstructive pulmonary disease: a reality or just a dogma? J Periodontol. 2013;84(12):1717-23. doi: 10.1902/ jop. 2013.120347 
13. Scannapieco FA, Ho AW. Potencial associations between chronic respiratory disease and periodontal disease: analysis of national health and nutrition examination survey III. J Periodontol. 2001 ;72(1):50-6. doi: 10.1902/ jop.2001.72.1.50

14. Zeng XT, Tu ML, Liu DY, Zheng D, Zhang J, Leng W. Periodontal disease and risk of chronic obstructive pulmonary disease: a meta-analysis of observational studies. Plos One. 2012 ; $7(10): e 46508$. doi: 10.1371 /journal.pone.0046508

15. Ledic K, Marinkovic S, Puhar I, Spali S, Popovic-Grle $\mathrm{S}$, Ivic-Kardum $\mathrm{M}$ et al. Periodontal disease increases risk of chronic obstructive pulmonary disease. Coll Antropol. 2013;37(3):937-42.

16. Wang Z, Zhou X, Zhang L, Song $Y$, Hu FB, Wang

C. Periodontal health, oral health behaviours, and chronic obstructive pulmonary disease. J Clin Periodontol. 2009;36(9):750-5. doi: $10.1111 / i .1600$ $\underline{051 X .2009 .01448 . x}$

17. Si Y, Fan H, Song $Y$, Zhou $X$, Zhang J, Wang Z. Association between periodontitis and chronic obstructive pulmonary disease in a chinese population. J Periodontol. 201 2;83(10):1 288-96. doi: 10.1902/iop.2012.110472

18. Deo V, Bhongade ML, Ansari S, Chavan RS. Periodontitis as a potential risk factor for chronic obstructive pulmonary disease: a retrospective study. Indian J Dent Res. 2009;20(4):466-70. doi: 10.4103/0970-9290.59456

19. Prasanna SJ. Causal relationship between periodontitis and chronic obstructive pulmonary disease. J Indian Soc Periodontol. 2011 ; 15(4):359-65. doi: 10.4103/0972$124 \times .92570$

20. Öztekin G, Baser U, Kucukcoskun M, Tanrikulu-Kucuk S, Ademoglu $E$, Isik $G$ et al. The association between periodontal disease and chronic obstructive pulmonary disease: a case control study. COPD. 2014;11(14):424-30. doi: 10.3109/15412555.2013.858316

21. Shen TC, Chang PY, Lin CL, Chen CH, Tu CY, Hsia $\mathrm{TC}$ et al. Risk of periodontal diseases in patients with chronic obstructive pulmonary disease: a nationwide population-based cohort study. Medicine (Baltimore). 2015;94(46):e2047. doi: 10.1097/ MD.0000000000002047

22. Chung JH, Hwang HJ, Kim SH, Kim TH. Associations between periodontitis and chronic obstructive pulmonary disease: the 2010 to 2012 Korean National Health and Nutrition Examination Survey. J Periodontol. 2016;87(8):86471. doi: 10.1902/iop.2016.150682

23. Bergström J, Cederlund K, Dahlén B, Lantz A-S, Skedinger $M$, Palmberg $L$ et al. Dental health in smokers with and without COPD. PLoS One. 2013;8(3):e59492. doi: 10.1371/journal. pone.0059492
24. Azarpapazhooh A, Leake JL. Systematic review of the association between respiratory diseases and oral health. J Periodontol. 2006;77(9):1465-82. doi: 10.1902/ jop. 2006.060010

25. Zhou X, Wang Z, Song Y, Zhang J, Wang C. Periodontal health and quality of life in patients with chronic obstructive pulmonary disease. Respir Med. $2011 ; 105(1)$ :67-73. doi: 10.1016/i.rmed.2010.06.017

26. Bhatt SP, Kim Y, Harrington KF, Hokanson JE, Lutz SM, $\mathrm{Cho} \mathrm{MH}$ et al. Smoking duration alone provides stronger risk estimates of chronic obstructive pulmonary disease than pack-years. Thorax. 2018;73(5):414:421. doi: 10.1136/ thoraxinl-2017-210722

27. Martinez FD. Early-life origins of chronic obstructive pulmonary disease. N Engl J Med. 2016;375(9):871-8. doi: 10.1056/NEJMra1603287

28. Barnes PJ. Inflammatory mechanisms in patients with chronic obstructive pulmonary disease. J Allergy Clin Immunol. 2016;138(1):16-27. doi: 10.1016/i.jaci.2016.05.011

29. Scott DA, Krauss J. Neutrophils in periodontal inflammation. Front Oral Biol. 2012;15:56-83. doi: $10.1159 / 000329672$

30. Nakayama $M$, Ohara N. Molecular mechanisms of Porphyromonas gingivalis-host cell interaction on periodontal diseases. Jpn Dent Sci Rev. 2017;53(4):134-140. doi: 10.1016/i.jdsr.2017.06.001

31. Shibata KI. Historical aspects of studies on roles of the inflammasome in the pathogenesis of periodontal diseases. Mol Oral Microbiol. 2018;33(3):203-211. doi: 10.1111/ omi.12217

32. Moeintaghavi A, Arab HR, Rezaee SAR, Naderi $H$, Shiezadeh F, Sadeghi $S$ et al. The effects of smoking on expression of IL- 12 and IL- $1 \beta$ in gingival tissues of patients with chronic periodontitis. Open Dent J. 2017;1 1:595-602. doi: $10.2174 / 1874210601711010595$

33. Bosshardt DD. The periodontal pocket: pathogenesis, histopathology and consequences. Periodontol 2000. 2018;76(1):43-50. doi: $10.1111 / \mathrm{prd} .12153$

34. Madalli $R$, Kheur S, Reddy MGS, Kheur M, Mahalle A. Assessment of role of Porphyromonas gingivalis as an aggravating factor for chronic obstructive pulmonary disease patients with periodontitis. Dent Hypotheses. 2016;7(3):1006. doi: $10.4103 / 2155-8213.190485$

35 Ji J, von Schéele I, Bergström J, Billing B, Dahlén B, Lantz AS et al. Compartment differences of inflammatory activity in chronic obstructive pulmonary disease. Respir Res. 2014;15:104. doi: 10.1186/s12931-014-0104-3 
36. Quadros AU, Dalposso LM, Karam TK, Mainardes RM, Khalil NM. Efeito da nicotina sobre fagócitos ativados. Acta Scientiarum. 2013;35(1):105-9. doi: $10.4025 /$ actascihealthsci.v35i1.11129

37. Katancik JA, Kritchevsky S, Weyant RJ, Corby P, Bretz $W$, Crapo RO et al. Periodontitis and airway obstruction. J Periodontol. 2005;76(supl 11):2161-7. doi: 10.1902/ jop.2005.76.11-S.2161

38. Barrionuevo AMP, Real FG, Igland J, Johannessen A, Omenaas $\mathrm{E}$, Franklin $\mathrm{KA}$ et al. Periodontal health status and lung function in two Norwegian cohorts. PLoS One. 2018;13(1):e0191410. doi: 10.1371/journal.pone.0191410

39. Bhavsar NV, Dave BD, Brahmbhatt NA, Parekh R. Periodontal status and oral health behavior in hospitalized patients with chronic obstructive pulmonary disease. J Nat Sci Biol Med. 2015;6(supl 1):S93-7. doi: 10.4103/09769668.166097

40. Macedo FR, Saba-Chujfi E, Pereira SAS, Costa EL, Melo Neto JP. Associação entre periodontite e doença pulmonar. RGO. $2010 ; 58(1): 47-53$.

41. Liu Z, Zhang W, Zhang J, Zhou X, Zhang L, Song $Y$ et al. Oral hygiene, periodontal health and chronic obstructive pulmonary disease exacerbations. J Clin Periodontol. 2012;39(1):45-52. doi: $10.1111 / \mathrm{i} .1600-$ $\underline{051 X .2011 .01808 . x}$

42. Takahashi T, Muro S, Tanabe N, Terada K, Kiyokawa $\mathrm{H}$, Sato $\mathrm{S}$ et al. Relationship between periodontitis-related antibody and frequent exacerbations in chronic obstructive pulmonary disease. PLoS One. $2012 ; 7(7):$ :40570. doi: 10.1371 /iournal.pone.0040570

43. Barros SP, Suruki R, Loewy ZG, Beck JD, Offenbacher S. A cohort study of the impact of tooth loss and periodontal disease on respiratory events among COPD subjects: modulatory role of systemic biomarkers of inflammation. PLoS One. 2013;8(8):e68592. doi: 10.1371//ournal. pone.0068592

44. Terashima T, Chubachi S, Matsuzaki T, Nakajima T, Satoh $M$, Iwami $E$ et al. The association between dental health and nutritional status in chronic obstructive pulmonary disease. Chron Respir Dis. 2017;14(4):334-41. doi: $10.1177 / 1479972316643076$

45. Kucukcoskun M, Baser U, Oztekin G, Kiyan E, Yalcin F. Initial periodontal treatment for prevention of chronic obstructive pulmonary disease exacerbations. J Periodontol. 2013;84(7):863-70. doi: 10.1902/iop.2012.120399
46. Zhou X, Han J, Liu Z, Song Y, Wang Z, Sun Z. Effects of periodontal treatment on lung function and exacerbation frequency in patients with chronic obstructive pulmonary disease and chronic periodontitis: a 2-year pilot randomized controlled trial. J Clin Periodontol. 2014;41(6):564-72. doi: 10.1111 /icpe. 12247

47. Shen TC, Chang PY, Lin CL, Chen CH, Tu CY, Hsia TC et al. Periodontal treatment reduces risk of adverse respiratory events in patients with chronic obstructive pulmonary disease: a propensity-matched cohort study. Medicine (Baltimore). 2016;95(20):e3735. doi: 10.1097/ MD.0000000000003735

48. Henke C, Budweiser S, Jorres RA. Lung function and associations with multiple dimensions of dental health: a prospective observational cross-sectional study. BMC Res Notes. 2016;9:274-82. doi: 10.1186/s13104-016-2079-2

49. Hyman JJ, Reid BC. Cigarette smoking, periodontal disease: and chronic obstructive pulmonary disease. J Periodontol. 2004;75(1):9-15. doi: 10.1902/jop.2004.75.1.9

50. Devlin J. Patients with chronic obstructive pulmonary disease: management considerations for the dental team. $\mathrm{Br}$ Dent J. 2014;217(5):235-7. doi: 10.1038/si.bdi.2014.756 